\title{
Anesthesia for Transurethral Resection of Bladder Tumors in Patients with Neuropathy Caused by Balloon- Occluded Arterial Infusion Treatment: A Retrospective, Observational Study
}

\author{
Junko Nakahira*, Shoko Nakano, Toshiyuki Sawai, Yu Miyazaki, Shunsuke Fujiwara and Toshiaki Minami \\ Department of Anesthesiology, Osaka Medical College, Japan
}

*Corresponding author: Junko Nakahira, Department of Anesthesiology, Osaka Medical College, Daigaku-machi, Takatsuki, Osaka, Japan

\begin{abstract}
Background: A novel bladder preservation therapy has been developed for patients with muscle-invasive bladder cancer called the Osaka Medical College (OMC) regimen. This regimen comprises balloon-occluded arterial infusion (BOAI) of an anticancer agent and with or without concurrent hemodialysis. Anticancer agents, such as cisplatin, can cause neurotoxicity, leading to peripheral neuropathy. This study aimed to investigate the occurrence of neuropathy after BOAI followed by a second transurethral resection of a bladder tumor (TURBT). We also aimed to investigate the effects of general and spinal anesthesia on neuropathy.
\end{abstract}

Methods: We performed a retrospective, observational study on 57 patients (49 men, 8 women, median age: 65 years) with bladder cancer who underwent a second TURBT after BOAI. TURBT was initially performed for diagnosis and then patients received the OMC regimen at 4-5 weeks after this first TURBT. Patients had general or spinal anesthesia for TURBT. For BOAI, an intra-arterial catheter with two occlusion balloons was used and inserted into the posterior trunk of the internal iliac artery. Cisplatin was infused through this catheter over 1 hour. Hemodialysis was simultaneously performed in patients $(\mathrm{n}=16)$ with a creatinine level $>1.5 \mathrm{mg} /$ dl. Radiation therapy was then performed in the pelvis. Patients underwent a second TURBT at 6 weeks.

Results: Seven of 57 patients had neuropathy. Symptoms caused by BOAI in the lower extremities were pain, numbness, and tingling. Although none of the treatments for neuropathy were particularly effective, the combination of tramadol and acetaminophen reduced tingling in one patient. No patients had adverse events of grade 3 or 4 . Two patients who had abnormal sensation before the second TURBT underwent general anesthesia to avoid any further deterioration.

Conclusion: This study showed that neuropathy occurs after BOAI of cisplatin for bladder cancer. Although there was no deterioration in sensation of the lower extremities in patients who had a second TURBT under general or spinal anesthesia, further deterioration in sensation is possible. Therefore, spinal anesthesia should be avoided in patients who have neuropathy due to BOAI.

Trial Registration: This study was registered in the Japanese Official Clinical Trial Registry (trial registration number: JMAIIA00171) and the registration date was 13 March 2014.

Keywords: Balloon occluded arterial infusion; Bladder cancer; Cisplatin; Neuropathy; Spinal Anesthesia; Transurethral resection of a bladder tumor

\section{Background}

Improvement of the survival rate in patients with bladder preservation required a new method that allows delivery of a high dosage of anticancer agent specifically into the tumor. A novel bladder preservation therapy in patients with muscle-invasive bladder cancer was developed in the Department of Urology in our institute. This therapy is called the Osaka Medical College (OMC) regimen. The regimen involves balloon-occluded arterial infusion (BOAI) of an anticancer drug with or without concurrent hemodialysis (HD). This procedure is then followed by radiation therapy. Cisplatin is used as an anticancer agent in the OMC 
regimen. Cisplatin in a nonprotein-bound form exerts its antitumor activity and dissipates quickly after administration. The half-life of cisplatin is normally less than one hour, and it decreases to below the detection limit four hours after injection [1,2]. Because the molecular weight of protein-unbound cisplatin is similar to that of creatinine, HD can provide efficient removal of cisplatin. Azuma et al. reported that approximately $95 \%$ of free Pt was removed by HD via the bilateral common iliac veins during BOAI. [3]. This can provide less toxicity into systemic circulation. A common side effect of cisplatin is neurotoxicity [4,5] leading to chemotherapy inducedperipheral neuropathy. This dose-dependent toxic neuropathy is associated with distal paresthesias, affecting the hands and feet, and often starts within 1 month of initiating cisplatin treatment and continues after cessation of the chemotherapy. Accumulation of cisplatin in dorsal root ganglion neurons in the form of platinumDNA adducts5 is thought to be one of the primary mechanisms of neurotoxicity [6]. We have found neuropathy in some patients after BOAI treatment. Although strong pain tended to disappear, tingling and numbness continued. Direct neurotoxic effects of cisplatin, small vessel injury from cisplatin, and blockage of blood flow cause neuropathy $[7,8]$. In the $\mathrm{OMC}$ regimen, transurethral resection of a bladder tumor (TURBT) is repeated 3-5 months after BOAI. TURBT is generally performed under spinal anesthesia because of Japanese health insurance regulations. The only exceptions are patients with designated reasons, such as anticoagulant therapy, previous spinal surgery, and necessity of neuromuscular blocking agents during surgery. Spinal anesthesia for a second TURBT might cause further deterioration of the patients' sensations. This study aimed to investigate the occurrence of neuropathy after BOAI followed by a second TURBT. We also aimed to investigate the effects of general and spinal anesthesia on neuropathy.

\section{Methods}

\section{Patients and Data Collection}

This retrospective observational study was approved by the Ethics Committee of Osaka Medical College, Japan (reference: 1357). The study was registered with the Japan Medical Association Center for Clinical Trials (reference: JMA-IIA00171) and registry URL is https://dbcentre3.jmacct.med.or.jp/JMACTR/App/ JMACTRE02_04/JMACTRE02_04.aspx?kbn=3\&seqno=4182). Data collection followed the STROBE statement. We reviewed the data of 57 patients who underwent TURBT for bladder cancer after BOAI in 2013 and 2014. Data were collected from the patients' clinical records and anesthetic charts. The anesthetic method for the second TURBT and postoperative changes in symptoms of neuropathy were reviewed.

\section{Anesthesia}

In patients who had general anesthesia for TURBT, anesthesia was induced with intravenous propofol $2 \mathrm{mg} / \mathrm{kg}$, rocuronium $0.8 \mathrm{mg} / \mathrm{kg}$, and an infusion of remifentanil at $0.5 \mu \mathrm{g} / \mathrm{kg} / \mathrm{min}$. A cuffed endotracheal tube or a subglottic device was inserted for airway management. Anesthesia was maintained with inhaled sevoflurane $1.0-1.5 \%$ and intravenous remifentanil $0.25-0.5 \mu \mathrm{g} / \mathrm{kg} / \mathrm{min}$ in a fraction of inspired oxygen of 0.4 . Sugammadex $1.5 \mathrm{mg} / \mathrm{kg}$ was administered intravenously to those who had received additional rocuronium during their surgical procedure. For postoperative analgesia, acetaminophen $1000 \mathrm{mg}$ was administered intravenously at the end of the surgery. All participants were encouraged to walk on the first postoperative day. In patients with spinal anesthesia, anesthesia was administered at L2/3, L3/4, or L4/5. Intrathecal bupivacaine hydrochloride (hyperbaric, $0.5 \%, 2.0-3.0 \mathrm{ml}$ ) was administered to achieve a T11 sensory level. All surgical procedures were performed using an electronic resectoscope with a monopolar view by surgeons. D-sorbitol 3\% was used as a non-conductive irrigation fluid, with the bags positioned $90 \mathrm{~cm}$ above the operating table. If the spinal anesthesia was ineffective, general anesthesia was started immediately.

\section{OMC Regimen and BOAI Therapy}

Patients underwent complete TURBT in order to be diagnosed. Patients received the OMC regimen at 4- 5 weeks after the first TURBT. All of the patients who received the OMC regimen had a neutrophil count of $1500 / \mu \mathrm{l}$, platelet count of $100,000 / \mu \mathrm{l}$, creatinine level of $3.0 \mathrm{mg} / \mathrm{dl}(265.2 \mu \mathrm{mol} / \mathrm{l})$ an Eastern Cooperative Oncology Group performance status of $0-2$, and no prior radiotherapy or systemic therapy for bladder cancer [9]. In order to perform intra-arterial infusion procedure, an intra-arterial catheter equipped with two occlusion balloons (size: 6 Fr, M6F-28-70-TBSB4-ST; Clinical Supply, Tokyo, Japan) was used. The catheter was inserted into the posterior trunk of the internal iliac artery through the femoral artery. After the distal balloon had passed through the bifurcation of the anterior trunk of the internal iliac artery, the distal and proximal balloons were inflated and fixed. As a result, the anterior trunk of the internal iliac artery, was isolated between the balloons. Using digital subtraction angiography, it was confirmed the tumor markedly stained because of active flow of injected contrast medium into the urinary bladder [10]. Cisplatin 50mg per each of the bilateral internal iliac arteries was locally infused through the catheter over 1 hour [11] (Figure 1). In patients with a creatinine level greater than $1.5 \mathrm{mg} / \mathrm{dl}$, HD was simultaneously performed via two double-lumen catheters (size: 12 Fr, Argyle $®$; Tyco Healthcare, Tokyo, Japan). These catheters were put in the bilateral common iliac veins for 2 hours after the beginning of arterial infusion. The catheters were connected to a hollow-fiber dialyzer (APS150; Asahi, Tokyo, Japan) with a membrane area of 1.0-1.5m2 [10]. 2-6 weeks after the OMC-regimen, radiation therapy was administered for a month to the whole pelvis for a total of 60Gy of local irradiation to the bladder. 6 weeks after radiation therapy, patients underwent a second transurethral resection of the site of the original tumor, ultrasound-guided whole-layer biopsy, and urine cytology. Their weight was recorded, and toxicity was monitored using the National Cancer Institute's Common Terminology Criteria for Adverse Events v4.0 (CTCAE) (Additional file 1). 


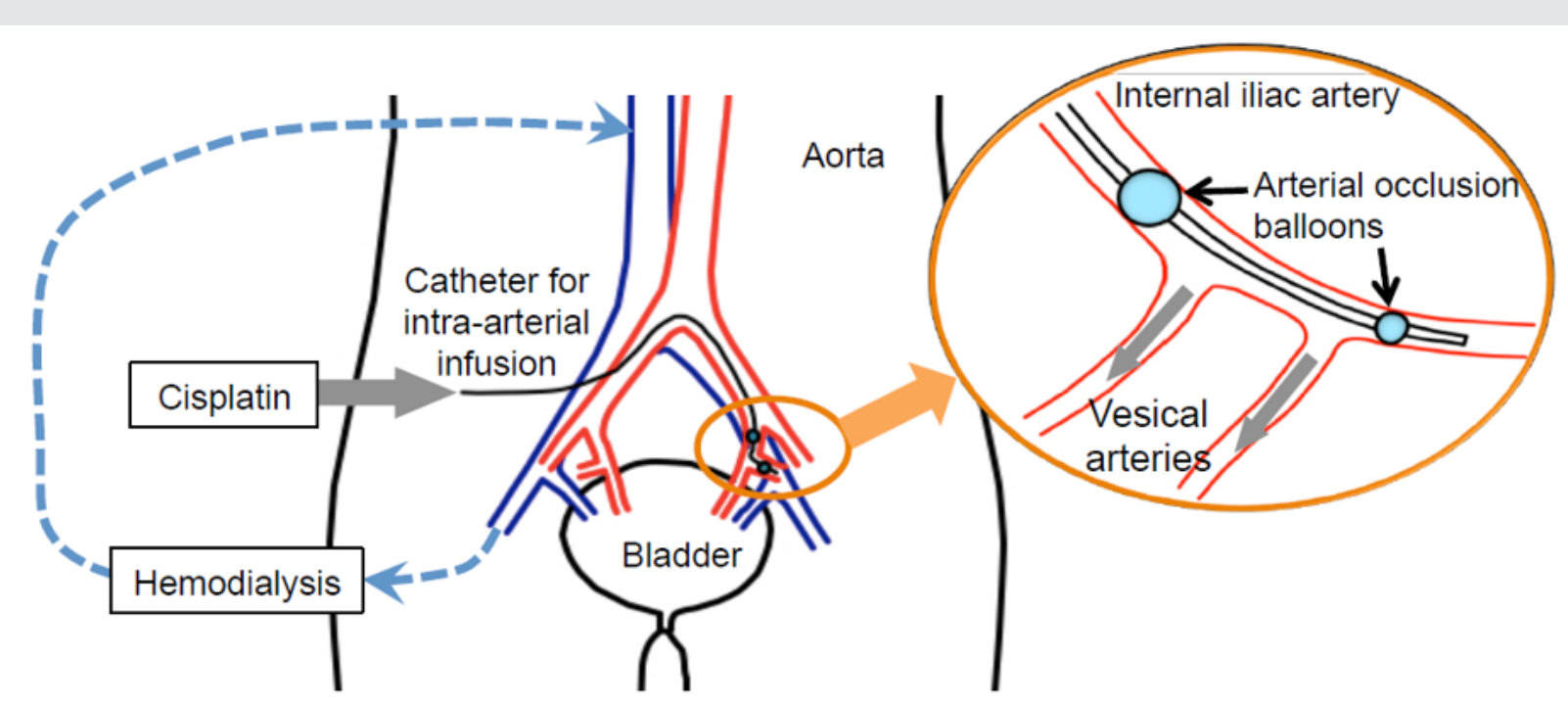

Figure 1: Schema of balloon-occluded arterial infusion therapy.

\section{Results}

A total of 57 patients underwent a second TURBT after BOAI. The median age of the patients was 65 years and the interquartile range was 60 to 72 years. Forty-nine patients were men and eight were women. Sixteen patients had HD and data were missing for two of 57 patients. None of the patients had adverse events of grade 3 or 4 . After radiation therapy, one patient had paralytic ileus and one had enteritis. Among the 57 patients, no patients showed skin ulcers or dermatitis caused by BOAI. None of the patients showed remarkably deteriorated renal function. None of the patients had hematological disorders after BOAI. Two patients had nausea and vomiting, and three patients had pollakiuria after BOAI. Seven of 57 patients had neuropathy at the preoperative anesthesia interview for the second TURBT. (Table 1) and (Figure 2) show the number of patients who received general or spinal anesthesia for the second TURBT, as well as neuropathy data. Among patients who underwent the second TURBT under spinal anesthesia, obturator nerve blocking was performed in 16 patients. of the 16 patients, three had ineffective nerve block and general anesthesia was started. Each case with neuropathy caused by BOAI is shown in (Table 1). Symptoms in the lower extremities caused by BOAI were pain, numbness, and tingling. Although none of the treatments, including pregabalin, were dramatically effective, the combination of tramadol and acetaminophen reduced tingling in patient [4]. This patient had TRAMSET® (Janssen Pharmaceutical K.K., Tokyo, Japan), which is a combination tablet of tramadol $37.5 \mathrm{mg}$ and acetaminophen 325mg, 3 or 4 times a day. Patients' 1 and 6 had pain and numbness and tingling in their lower extremities before treatment. Patient 6 had herniation in his lumber vertebra. These two patients had already taken loxoprofen before the entire treatment. These two patients underwent a second TURBT under general anesthesia to avoid deterioration of sensation.

Table 1: ASA-PS; American Society of Anesthesiologists Physical Status, BOAI; balloon-occluded arterial infusion, TURBT; transurethral resection of a bladder tumor.

\begin{tabular}{|c|c|c|c|c|c|c|c|c|}
\hline Case & Age & Sex & ASA-PS & $\begin{array}{c}\text { Hemodyalysis } \\
\text { during BOAI }\end{array}$ & $\begin{array}{c}\text { Location of } \\
\text { neuropathy }\end{array}$ & Symptoms & $\begin{array}{c}\text { Treatment for } \\
\text { neuropathy }\end{array}$ & $\begin{array}{c}\text { Anesthesia for the } \\
\text { second TURBT }\end{array}$ \\
\hline 1 & 73 & Male & 3 & No & Both legs & $\begin{array}{c}\text { Numbness, tingling, } \\
\text { and pain }\end{array}$ & Loxoprofen \\
\hline 2 & 63 & Male & 2 & No & Both legs & Numbness, tingling & None & Spinal \\
\hline 3 & 64 & Male & 3 & No & Left leg & $\begin{array}{c}\text { Numbness, tingling, } \\
\text { and pain }\end{array}$ & $\begin{array}{c}\text { Loxoprofen, } \\
\text { pregabalin }\end{array}$ \\
\hline 4 & 62 & Male & 2 & No & Both legs & $\begin{array}{c}\text { Numbness and } \\
\text { tingling }\end{array}$ & $\begin{array}{c}\text { Pregabalin, tramadol, } \\
\text { acetaminophen }\end{array}$ \\
\hline 5 & 24 & Male & 1 & No & Left leg & $\begin{array}{c}\text { Numbness and } \\
\text { tingling }\end{array}$ & None \\
\hline 6 & 45 & Female & 2 & No & Both legs & $\begin{array}{c}\text { Numbness and } \\
\text { tingling }\end{array}$ & Pregabalin & Spinal \\
\hline 7 & 64 & Male & 3 & Yes & Both legs & Pain & Pregabalin & General \\
\hline
\end{tabular}




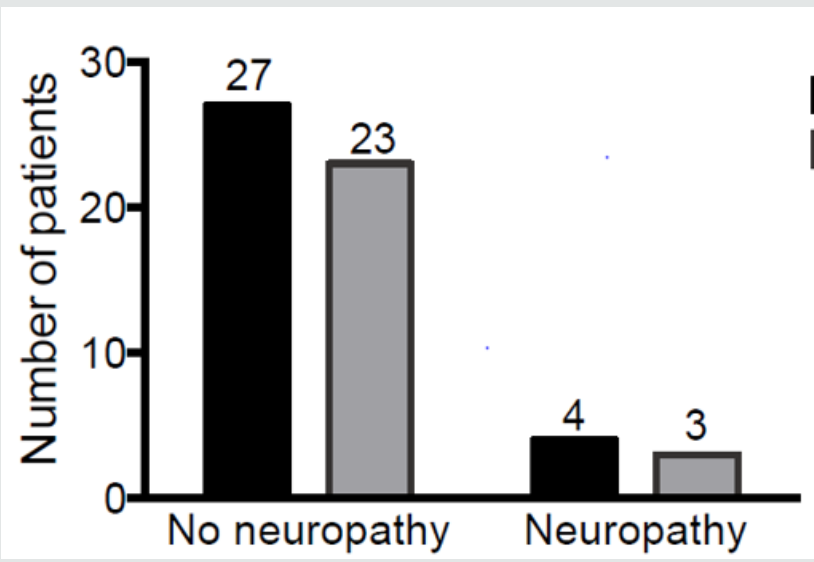

Figure 2: Results of anesthesia types for the second transurethral resection of a bladder tumor. The overview of the patients who had neuropathy after BOAI and the anesthesia types for the second transurethral resection of a bladder tumor was shown.

\section{Discussion}

In this study, the symptoms of neuropathy caused by BOAI were limited to pain, tingling, and numbness. Some patients complained of a burning sensation with strong tingling. The location of the abnormal sensation was limited to the lower extremities, including the gluteal region, femora, crus, and planta. Although the most concerning complications caused by BOAI are severe hematological, genitourinary, and gastrointestinal toxicity, neuropathy is not lifethreatening. However, long-term numbness, pain or tingling can reduce the patients' quality of life. In the two patients who had abnormal sensation before treatment, general anesthesia was performed instead of spinal anesthesia. There was the possibility that the patients would complain about an increase in abnormal sensation after spinal anesthesia at the postoperative interview 24 hours after the second TURBT. However, anesthesiologists monitored postoperative recovery of the patients to check for deteriorated sensation and did not find any deterioration. BOAI for bladder cancer via the internal iliac artery is widely spreading in use among hospitals worldwide as a treatment for cancer. This is because this procedure can deliver anticancer drugs with a high concentration only to the bladder. Embolization in the arteries of the lower extremities, ulcers and dermatitis, and sciatic neuralgia have been reported as adverse effects of BOAI [8,12-14]. Nerve injuries caused by intra-arterial infusion of cisplatin are a direct neurotoxic effect of cisplatin and chemotherapy-induced small vessel injury, with subsequent plexus or nerve infarction [7]. Sensory nerves are usually damaged [8]. BOAI has the potential to cause nerve injury at a high rate because the remaining cisplatin spreads to distant arteries after the release of blood supply. HD can reduce the possibility of neuropathy occurring. However, our study lacks the data to conclude that HD reduced neuropathy. In addition, blockage of blood supply is the main reason for the occurrence of neuropathy [8]. Blood supply to the lower extremities was stopped for longer than 1 hour in our present study and it would have caused acute neural damage.

\section{General anesthesia $\square$ Spinal anesthesia}

The branches from the internal iliac artery supply blood to the cauda equina and the nerves that enter the pelvis. The arteria comitans nervi ischiadici arises from the inferior vesical artery and it supplies blood to the pelvic region of the sciatic nerve. The spinal branches form the lateral sacral artery supply blood to the spinal cord and nerve root of the sciatic nerve. The iliacus branch of the iliolumbar artery supplies blood to the femoral nerve and the lumbar branch of the iliolumbar artery supplies blood to the cauda equine. An anticancer agent coming from the inferior gluteal artery, which is an anterior division of the internal iliac artery, to the sciatic nerve would cause damage of the sciatic nerve during BOAI from the internal iliac artery. Therapy for neuropathy includes loxoprofen, pregabalin, and the combination of tramadol and acetaminophen. The combination of tramadol and acetaminophen was effective in one of our patients, although the neuropathy did not completely disappear. Tramadol binds to the $\mu$-opioid receptor and inhibits uptake of serotonin and norepinephrine. Tramadol could be effective for other patients with neuropathy caused by BOAI. This study had some limitations. One limitation was the small sample size. Abnormal sensations were changeable and vague in some patients. We did not use the Numerical Rating Scale, Visual Analogue Scale, Verbal Rating Scale, or Support Team Assessment Schedule. Furthermore, the method of anesthesia for the second TURBT was determined by anesthesiologists, surgeons, and patients. Even if the patients had neuropathy after BOAI, the patients tended to receive spinal anesthesia because they experienced spinal anesthesia for the first TURBT. In those cases, the sensations after spinal anesthesia for the second TURBT did not worsen. However, anesthesiologists should explain the possibility of deterioration of sensations in the lower extremities. Although general anesthesia costs more than spinal anesthesia, we consider that spinal anesthesia should be avoided for a second TURBT after BOAI. Patients with bladder cancer tend to have repeated TURBT. Therefore, the most appropriate anesthesia should be determined and explained to these patients. 


\section{Conclusion}

This study showed that neuropathy occurred in the lower extremities after BOAI of cisplatin for bladder cancer. BOAI caused neuropathy, with symptoms of numbness, tingling and pain. Although there was no deterioration in sensation of lower extremities in patients who had a second TURBT under general or spinal anesthesia, there was a possibility of further deterioration in sensation. Spinal anesthesia should be avoided in patients who have neuropathy caused by BOAI.

\section{Ethics Approval and Consent to Participate}

The Ethics Committee of Osaka Medical College approved the study protocol (reference number: 1357), which waived the requirement for informed consent because of the retrospective design of the study.

\section{Authors' Contributions}

Junko Nakahira designed and carried out the study and drafted the manuscript. Shoko Nakano collected the data and helped to draft the manuscript. Toshiyuki Sawai participated in the design of the study and performed the statistical analysis. Yu Miyazaki and Shunsuke Fujiwara helped to draft the manuscript. Toshiaki Minami conceived the study, participated in its design and coordination, and helped to draft the manuscript. All authors read and approved the final manuscript.

\section{References}

1. Stöckle M, Wellek S, Meyenburg W, Voges GE, Fischer U, et al. (1996) Radical cystectomy with or without adjuvant polychemotherapy for non-organ-confined transitional cell carcinoma of the urinary bladder: prognostic impact of lymph node involvement. Urology 48(6): 868-875.

2. Herr HW, Bochner BH, Dalbagni G, Donat SM, Reuter VE (2002) Impact of the number of lymph nodes retrieved on outcome in patients with muscle invasive bladder cancer. J Urol 167(3): 1295-1298.
3. Azuma H, Kotake Y, Yamamoto K, Sakamoto T, Kiyama S (2008) Effect of combined therapy using balloon-occluded arterial infusion of cisplatin and hemodialysis with concurrent radiation for locally invasive bladder cancer. Am J Clin Oncol 31(1): 11-21.

4. Von Hoff DD, Schilsky R, Reichert CM, Reddick RL, Rozencweig M (1979) Toxic effects of cis-dichlorodiammineplatinum(II) in man. Cancer Treat Rep 63(9-10): 1527-1531.

5. Mollman JE (1990) Cisplatin neurotoxicity. N Engl J Med 322(2): 126127.

6. Zhu J, Carozzi VA, Reed N, Mi R, Marmiroli P, et al. (2016) Ethoxyquin provides neuroprotection against cisplatin-induced neurotoxicity. Sci Rep 6: 28861.

7. Castellanos AM, Glass JP, Yung WK (1987) Regional nerve injury after intra-arterial chemotherapy. Neurology 37(5): 834-837.

8. Nagamatsu H, Matsumura T, Wakui M (1993) Sciatic nerve paralysis following intraarterial chemotherapy for bladder cancer. Acta Urol Jpn 39(8): 743-746.

9. Azuma H, Inamoto T, Ibuki N, Ubai T, Kotake Y (2010) Novel bladder preservation therapy for locally invasive bladder cancer: combined therapy using balloon-occluded arterial infusion of anticancer agent and hemodialysis with concurrent radiation. Int J Oncol 37(4): 773-785.

10. Azuma H, Inamoto T, Takahara K, Nomi H, Uehara H (2013) Effect of a novel bladder preservation therapy, BOAI-CDDP-radiation (OMCregimen). Int J Oncol 43(1): 79-87.

11. Yoshioka H, Shimbo T, Yoshida K, Yoshikawa N, Uesugi Y, et al. (2016) Treatment results of radiotherapy combined with balloon-occluded arterial infusion chemotherapy for invasive bladder cancer. Anticancer Res 36(2): 731-736.

12. Wallace S, Chuang VP, Samuels M, Johnson D (1982) Transcatheter intraarterial infusion of chemotherapy in advanced bladder cancer Cancer 49(4): 640-645.

13. Kanoh S, Umeyama T, Nemoto S, Ishikawa S, Nemoto R, et al. (1983) Long-term intra-arterial infusion chemotherapy with adriamycin for advanced bladder cancer. Cancer Chemother Pharmacol 11: S51-S58.

14. Kato T, Nemoto R, Mori H, Takahashi M, Tamakawa Y (1981) Arterial chemoembolization with microencapsulated anticancer drug. An approach to selective cancer chemotherapy with sustained effects. JAMA 245(11): 1123-1127
To Submit Your Article Click Here:

This work is licensed under Creative Commons Attribution 4.0 License

DOI: $10.32474 /$ GJAPM.2019.01.000104

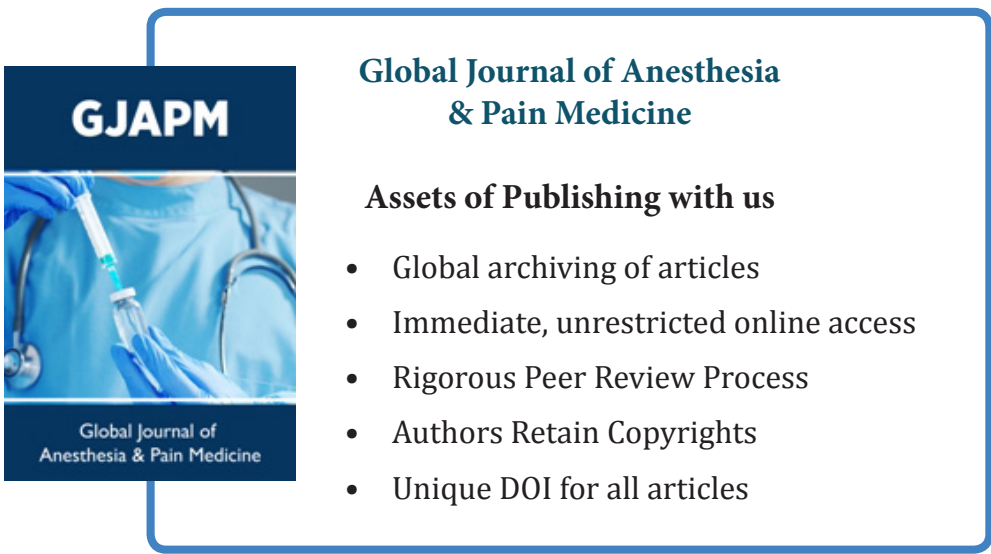

